In carrying out this work we have been greatly helped by the co-operation of the Research Lab. oratories of Messrs. Kodak, Ltd., Harrow.

E. J. G. BeEson

Research Laboratory,

British Thomson Houston Co., Ltd., Rugby. March 24.

\section{Novel Methods of Producing Edge Tones with Liquids}

WE have recently observed what would appear to be two new methods of producing vortex edge tones (a) by allowing jets of water to impinge on perforated diaphragms and $(b)$ by rotating perforated metallic disks under water.

(a) Singing perforated diaphragms excited by liquid jets. If a jet of water is played almost tangentially on a perforated diaphragm, then, under suitable conditions, the diaphragm is set into vibration and emits a loud audible note.

A convenient arrangement consists of a perforated disk of zinc, $0.3 \mathrm{~mm}$. thick, soldered to one end of a brass tube of 5-10 centimetres diameter. The perforations can be 'close packed' $\mathbf{1 . 4}$ millimetres in diameter and with five holes to the centimetre. Water flowing through a rubber tube impinges on the diaphragm at a speed roughly controlled by pinching the tube and in a direction approximately tangential to the diaphragm.

The tones elicited vary somewhat with the position and angle of impact,but seem to bear no simple relation to the fundamental frequency of the loaded diaphragm. Adjustments are not critical and it is not necessary for the diaphragm to be perfectly flat. Occasionally two distinct notes are produced simultaneously. Using the dimensions stated above, the frequencies of the notes produced varied from below 500 to more than 1,200 . Some reinforcement of the tones is obtained by using a resonating air column; but the resonance is usually not very pronounced.

(b) Edge tones produced by rotation of a perforated disk immersed in a liquid. When a thin solid flat metallic disk is rapidly rotated under water about an axis perpendicular to its plane, there is usually no production of audible sound. If, however, the disk is perforated with a ring of similar regularly distributed holes, then, at certain speeds of rotation, audible sounds characteristic of the speed can be heard with the aid of a submerged listening device.

The rotating disk does not behave as a siren, since the notes it produces are separated by silent intervals as the speed of rotation is varied. Usually the frequency of the emitted note is higher at high speeds of rotation, but occasionally the same note or even a lower note is produced as the speed is increased.

Suitable disks for producing these effects may be of brass or aluminium, $1 \mathrm{~mm}$. thick, $10-15 \mathrm{~cm}$. in diameter, perforated with holes of $3 \mathrm{~mm}$. diameter. A single ring of 9 or 12 equally spaced holes or concentric rings of the same number of holes with ring diameters from $5-10 \mathrm{~cm}$. have been found effective. For a given range of speeds more notes are produced with the multiple perforated ring system than with a single ring system.

Physics Department, Medical College of

\section{F. L. HoPwooD}

St. Bartholomew's Hospital,

London, E.C.J. July 29.

\section{Equilibrium of Ionization in the Lower Atmosphere}

ONE of the problems of atmospheric electricity is to account for the relation between the concentration of small ions near the ground and the number of Aitken condensation nuclei present. Small ions are lost in two ways, by recombination among themselves and by attachment to the Aitken nuclei. When nuclei are plentiful, the first process is relatively unimportant. If we assume a constant rate of production of $q$ ion pairs $/ \mathrm{cm}^{3} / \mathrm{sec}$, any straightforward type of theory predicts a relation of the type $q=n Z \times$ const., where $n$ is the concentration of small ions of one sign and $Z$ is the concentration of nuclei. So far is this from being true that formule of the type $q=\xi_{n} Z^{1 / 2} 1$ and $n Z^{1 / 5}=$ const. ${ }^{2}$ have been proposed to fit the experimental results.

Some observations made near Dublin between January and July 1948 on the radium A content of atmospheric air, with simultaneous observations of nucleus content, may throw some light on the problem.

\begin{tabular}{|c|c|c|c|c|c|}
\hline Nuclei $/ \mathrm{cm}^{3}$ & $<5,000$ & $\begin{array}{l}5,000- \\
10,000\end{array}$ & $\begin{array}{l}10,000- \\
20,000\end{array}$ & $\begin{array}{c}20,000- \\
30,000\end{array}$ & $>30,000$ \\
\hline $\begin{array}{l}\text { Ra A atoms } \times \\
10^{-5} / \mathrm{cm}^{3} \\
\text { No. of observations }\end{array}$ & $\begin{array}{l}7 \cdot 4 \\
67\end{array}$ & $\begin{array}{r}7 \cdot 5 \\
152\end{array}$ & $\begin{array}{l}10 \cdot 1 \\
139\end{array}$ & $\begin{array}{c}15 \cdot 5 \\
32\end{array}$ & $\begin{array}{c}27 \cdot 3 \\
21\end{array}$ \\
\hline
\end{tabular}

With increasing concentration of nuclei, there is a marked increase in radium A content. It seems clear that the conditions which favour the accumulation of one kind of body near the ground favour that of the other. According to Hess ${ }^{3}$, about half the ionization at ground-level is due to the radioactive matter in the air. The position is that the rate of ion-production in any place where nuclei are plentiful is far from being constant, but increases with the increase of nuclei. Any simple form of equilibrium relation between $n$ and $Z$ is, therefore, not to be expected.

J. J. NOLAN

University College,

T. BURKE Dublin.

March 30.

${ }^{1}$ Nolan, P. J., Proc. Roy. Irish Acad., 38, 49 (1929).

'Wright, H. L., Quart. J. Roy. Met. Soc., 61, 93 (1935).

${ }^{3}$ Hess, V. F., "The Electrical Conductivity of the Atmosphere" (Constable, 1928)

\section{High-Frequency Discharges Localized Along Tracks of Ionizing Particles}

IT has been found that an intense high-frequency electric field can produce visible discharges localized along the tracks of $\alpha$-particles and $\beta$-particles passing through a gas.

A closed glass tube $A$ provided with a thin mica window $B$ was fitted into a wire gauze cylindrical wave-guide $C$ which was excited in the $T E$, mode by $1.4 \mu$ sec. pulses from a $60-\mathrm{kW}$. peak power 3,000-Mc./s. magnetron. The power delivered to this section could be varied, and standing waves were eliminated by matching the absorbing termination of the wave guide.

The photographs were obtained with an uncorrected condenser lens ( $f$ number $\sim 0 \cdot 6$ ), and the tube was filled with neon saturated with iodine vapour at a total pressure of about $20 \mathrm{~cm}$. mercury. In the 